\title{
Fatores associados ao desenvolvimento de úlceras de pressão: o impacto da nutrição
}

\author{
Factors associated with the development of \\ pressure ulcers: the impact of nutrition
}

Suellen Fabiane CAMPOS ${ }^{1}$

Ângela Conceição Pereira CHAGAS'

Aline Bárbara Pereira COSTA ${ }^{1}$

Rosilene Estevão de Melo FRANÇA²

Ann Kristine JANSEN ${ }^{1}$

RE S U M O

Objetivo

Trata-se de um estudo prospectivo com o objetivo de determinar a incidência, em um hospital universitário, de úlceras de pressão e verificar fatores nutricionais envolvidos em seu desenvolvimento.

\section{Métodos}

Foram registrados dados clínicos, sociodemográficos, antropométricos e dietéticos de 50 pacientes, posteriormente analisados com auxílio do programa Epi Info 3.2.

\section{Resultados}

Verificou-se a predominância de indivíduos do sexo feminino, com média de idade de $66,6, \mathrm{DP}=18,0$ anos. A incidência das injúrias foi de 28,0\%, resultando em uma média de 1,7 feridas por paciente, mais frequentes na região sacral $(57,1 \%)$ e em calcanhares $(35,7 \%)$. Encontrou-se significância estatística $(p<0,05)$ para as variáveis: mudança de decúbito, nível de atividade e uso de fralda, assim como para a presença de doenças mentais, neurológicas, anemia, uso de antibióticos, anti-inflamatórios e imunossupressores. Com relação aos fatores nutricionais, foi encontrada associação positiva entre pacientes com úlcera e baixos valores da área muscular do braço, circunferência de panturrilha, pregas cutâneas tricipital e subescapular, hemoglobina, hematócrito e valores elevados de leucócitos. As feridas também estiveram associadas a maiores índices de mortalidade e ao aumento do tempo de internação.

\section{Conclusão}

Observou-se que um estado nutricional deficiente está estreitamente relacionado com o desenvolvimento de úlceras de pressão. Neste estudo, foram identificados alguns parâmetros nutricionais relacionados com esse evento que poderão ser ferramentas importantes na identificação e no tratamento de pacientes em risco.

Termos de indexação: Estado nutricional. Hospitalização. Úlcera de pressão.

1 Universidade Federal de Minas Gerais, Escola de Enfermagem, Curso de Nutrição. Av. Prof. Alfredo Balena, 190, Santa Efigênia, 30130-100, Belo Horizonte, MG, Brasil. Correspondência para/Correspondence to: S.F. CAMPOS. E-mail: <susufabi@yahoo.com.br>.

2 Nutricionista, Hospital Universitário Risoleta Tolentino Neves. Belo Horizonte, MG, Brasil. 
704 | S.F. CAMPOS et al.

\section{A B S T R A C T}

\section{Objective}

This prospective study aimed to determine the incidence of pressure ulcers in a university hospital and investigate the nutritional factors involved in their development.

\section{Methods}

The clinical, sociodemographic, anthropometric and dietary data of 50 patients were collected and analyzed by the software Epi Info 3.2.

\section{Results}

Females aged $M=66.6, S D=18.0$ years prevailed. The incidence of pressure ulcers was $28.0 \%$, resulting in roughly 1.7 pressure ulcers per patient. The most common sites were the sacral region (57.1\%) and heels $(35.7 \%)$. Statistical significance $(p<0.05)$ was found for the following variables: changing position in bed, activity level, use of diaper, mental or neurological illness, anemia and use of antibiotics, anti-inflammatories and immunosuppressants. With respect to nutritional factors, positive associations were found between patients with ulcers and low arm muscle area, low calf circumference, low triceps and subscapular skinfold thicknesses, low hemoglobin level, low hematocrit, and high white blood cell count. The wounds also were associated with higher mortality rates and longer hospital stays.

\section{Conclusion}

There is a close association between poor nutritional status and the development of pressure ulcers. This study identified some nutritional parameters related to this event which may become important tools for the identification and treatment of patients at risk.

Indexing terms: Nutritional status. Hospitalization. Pressure ulcer.

\section{N T R O D U ÇÃ O}

Muitos termos são usados como sinônimos para Úlcera de Pressão (UP), dentre eles escara, úlcera de decúbito e ferida por pressão $0^{1,2}$. A UP é definida como uma injúria localizada na pele e no tecido subjacente, que usualmente ocorre sobre proeminências ósseas, como cotovelos, tornozelos e quadris, ou áreas que recebem grande quantidade de pressão ${ }^{1-10}$. A compressão do tecido sensível sobre a proeminência óssea resulta em subsequente redução do fluxo sanguíneo e isquemia do local, causando hipóxia dérmica, necrose, ruptura da epiderme e eventualmente contaminação bacteriana 2,11,12.

A prevalência desse evento varia entre 3\% e $66 \%$, de acordo com as condições do paciente, a doença e o tipo de instituição onde o paciente é tratado $1,2,4,5,8,13-19$. No ambiente hospitalar, a prevalência varia de 5 a $40 \%$ 4,13,15,18,20. O desenvolvimento de UP implica sobrecargas físicas, emocionais e sociais para os pacientes e suas famílias, reduzindo, consequentemente, a quali- dade de vida e aumentando os custos dos serviços de saúde, ocasionados pelos longos períodos de hospitalização e maiores índices de morbidade e mortalidade ${ }^{1-3,7,8,12} 17$.

A etiologia da UP é multifatorial e depende de fatores de risco extrínsecos e intrínsecos. Estudos apontam como fatores extrínsecos forças de fricção e cisalhamento e, como intrínsecos, idade avançada (>80 anos), sexo, limitação nas atividades de vida diária, incontinência urinária e ou anal, anemia, infecção e estado nutricional1,4,7,21-23. Outros fatores de risco citados são: uso de alguns tipos de medicamento, nível de consciência e percepção sensorial reduzidos, história prévia de UP, estado mental depletado, imobilidade e prejuízo do sistema imune ${ }^{2-6,8,8,12,16,17,21,23}$.

Na prática diária, muitos profissionais referem-se à desnutrição como um dos principais fatores que contribuem para UP; eles relatam que a úlcera é um expoente da fragilidade interna e têm observado que pacientes com UP ou com risco aumentado para desenvolvê-la frequentemente não estão nutridos de forma adequa$\mathrm{da}^{3,16,21}$ 
Dentre os fatores nutricionais que interferem no risco para o desenvolvimento de UP estão: inadequação da ingestão dietética, pobre ingestão habitual de proteína, baixo índice de massa corporal, perda de peso, valores baixos de pregas cutâneas e baixos níveis séricos de albumina $^{3,6,16,17,23}$.

Apesar de a literatura citar a estreita relação da nutrição com o desenvolvimento das úlceras de pressão, este trabalho traz como inovação o aprofundamento do estudo da relação entre UP e variáveis antropométricas e dietéticas a fim de que mais informações estejam disponíveis e que possa haver uma otimização tanto da prevenção quanto do tratamento dessas feridas.

O presente estudo teve como objetivo determinar a incidência de úlceras de pressão em um hospital universitário e verificar fatores nutricionais e clínicos envolvidos no seu desenvolvimento.

\section{M É T O D O S}

Estudo prospectivo desenvolvido em um Hospital Universitário, localizado em Belo Horizonte, MG, aprovado pelo Comitê de Ética da Universidade Federal de Minas Gerais (parecer n 642/07), e autorizado pelo Núcleo de Ensino, Pesquisa e Extensão do referido hospital.

A amostra foi calculada a partir da prevalência de úlcera de pressão, estimada em $40 \%{ }^{18}$, e do número de internações ocorrido nos quatro meses anteriores à coleta de dados nos setores do hospital envolvidos no estudo ( $n=1$ 208). Considerou-se precisão de $5 \%$ e intervalo de confiança de $95 \%$, resultando em uma amostra mínima de 40 indivíduos, à qual se acrescentou 20\% para compensar possíveis perdas, totalizando 48 indivíduos. Para o cálculo foi utilizado o programa STATCALC do softwear Epi Info 6.04.

Foram selecionados todos os pacientes internados na clinica médica ou no Centro de Terapia Intensiva (CTI) que atendessem os seguintes critérios de inclusão: ser maior de 18 anos; não apresentar queimaduras de ampla extensão; estar internado por menos de 48 horas e apresentar o Termo de Consentimento Livre e Esclarecido assinado.

Os demais setores da instituição - maternidade, pronto socorro e clínica cirúrgica - foram excluídos por apresentarem grande rotatividade de pacientes, o que poderia implicar tempo insuficiente de internação para observação do evento e grande perda de participantes ao longo do estudo.

Excluíram-se pacientes que apresentaram alguma dificuldade para responder ao questionário e não tinham acompanhante; menores de 18 anos; que haviam sido reinternados.

A amostra final foi composta por 50 pacientes: 9,6\% das 519 internações que ocorreram no período de coleta dos dados. Três pesquisadoras foram responsáveis pela coleta de dados, avaliações antropométricas e observação quanto ao aparecimento das úlceras de pressão.

Quanto à UP, quando presente, foi detectada mediante observação direta da ferida ou verificação de registro referente a ela no prontuário médico ou da equipe de enfermagem, seguida da observação visual das pesquisadoras. Não foi avaliado o estágio da ferida.

A fim de avaliar a incidência da UP na instituição, sem criar viés por mudança de conduta, os profissionais envolvidos no processo de cuidado dos pacientes, com exceção das nutricionistas, não tinham conhecimento quanto aos pacientes selecionados.

Foram registrados, do prontuário médico e da equipe de enfermagem, tempo de internação, dados clínicos referentes ao diagnóstico principal, comorbidades, resultados de exames, medicação utilizada, uso de fralda, assim como a prescrição de mudança de decúbito e o número de dias em que essa recomendação foi registrada. Informações sociodemográficas, como idade, sexo, cor de pele, renda, escolaridade e presença de acompanhante, foram coletadas por meio do questionário próprio padronizado. 
Os dados antropométricos foram coletados, semanalmente, seguindo as recomendações da Organização Mundial da Saúde ${ }^{24}$. Para avaliação física dos pacientes foram aferidas as Pregas Cutâneas Tricipital (PCT), Bicipital (PCB) e Subescapular (PCSE) com uso de adipômetro da marca Lange (EUA); e as Circunferências de Braço (CB) e Panturrilha (CP) com fita métrica flexível e inelástica, de $150 \mathrm{~cm}$ de extensão e $0,1 \mathrm{~cm}$ de precisão. As dobras foram aferidas três vezes, sendo registrado somente o valor médio.

Foi calculada também a Área Muscular do Braço corrigida $\left(A M B_{c}\right)$, utilizando-se fórmula proposta por Gurney \& Jelliffe ${ }^{25}$.

Os pacientes foram avaliados três vezes por semana quanto ao aparecimento de UP e de novas comorbidades. Os dados referentes aos resultados de exames de glicose e hemograma foram coletados no início da internação e após trinta dias. No caso dos pacientes que receberam alta em período inferior a um mês, foram registrados somente os resultados referentes à internação. Os registros dos medicamentos prescritos e das possíveis alterações na prescrição foram realizados semanalmente. Todos os medicamentos prescritos foram considerados na análise, independentemente do tempo de uso e do momento de prescrição.

O diagnóstico principal e as comorbidades foram classificadas conforme a Classificação Internacional de Doenças (CID ${ }^{26}$. Os medicamentos prescritos foram agrupados segundo sua função $0^{27,28}$.

Para avaliar a ingestão dietética dos pacientes que se alimentavam por via oral, foram aplicados três recordatórios 24 horas, em dias alternados, na primeira semana de internação. Posteriormente, a frequência de aplicação desse instrumento foi semanal. Quando o paciente não tinha condições de responder ao recordatório, o acompanhante assumia essa função.

Para os pacientes em uso de nutrição enteral, registrou-se, em três dias alternados, na primeira semana de internação, o volume diário de dieta infundida. Essa informação foi coletada do prontuário da enfermagem ou da nutrição. O volume infundido foi reavaliado semanalmente.

O cálculo dos nutrientes ingeridos foi realizado a partir dos dados da Tabela Brasileira de Composição de Alimentos ${ }^{29}$ e das informações nutricionais presentes nos rótulos dos suplementos e das dietas enterais industrializadas.

$\mathrm{Na}$ análise dos dados, os pacientes foram divididos em dois grupos: um formado por aqueles que desenvolveram úlcera de pressão em algum momento da internação, e outro por aqueles que não desenvolveram as feridas. O banco de dados foi analisado no programa Epi Info versão 3.2. A análise descritiva das variáveis foi realizada mediante cálculo das distribuições de frequências, média e desvio-padrão. Analisaram-se os fatores associados à presença de UP, por meio do teste qui-quadrado de Pearson ou do teste exato de Fisher, para as variáveis categóricas. Para as contínuas, foi utilizado o teste de Mann-Whitney. O nível de significância menor que 5,0\% foi adotado para todos os testes.

\section{RES ULTA DOS}

O estudo foi realizado nos meses de abril e maio de 2008 , obtendo-se uma amostra final de 50 indivíduos. Os pacientes foram acompanhados até a alta hospitalar, o óbito ou até o encerramento da pesquisa, por um período médio de 10,4, Desvio-Padrão (DP)= 8,2 dias (1 - 36 dias).

O intervalo entre a internação e a $1^{\text {a }}$ avaliação foi em Média (M) de 36,9, DP=11,9 horas (5 - 48 horas). Quanto à unidade em que os indivíduos estavam alocados, 90,0\% ( $n=45)$ eram pacientes da clínica médica, 4,0\% ( $n=2)$, provenientes do centro de terapia intensiva, e o restante $(6,0 \%)$ oscilou entre as duas unidades.

Em relação aos dados sociodemográficos, $68,0 \%$ dos pacientes eram do sexo feminino, $60,0 \%$ foram classificados como pardos ou negros e mais da metade da população $(61,1 \%)$ foi composta por maiores de 60 anos. A média de idade 
foi de $66,6 \mathrm{DP}=18,0$ anos (24-104 anos). Contudo, não houve diferença estatisticamente significativa $(p>0,05)$ para essas variáveis entre os indivíduos com e sem úlcera $M=72,93, D P=14,04$ e $M=64,14, D P=18,94$, respectivamente). $O$ mesmo foi observado em relação à renda per capita ( $M=0,9, D P=1,2$ e $M=0,8, D P=0,4$ salário-míni$\mathrm{mo}^{(3)}$ respectivamente) e escolaridade $(\mathrm{M}=2,9$, $\mathrm{DP}=2,5 \mathrm{M}=3,2, \mathrm{DP}=3,1$ anos respectivamente).

A incidência de UP nos participantes da pesquisa foi de $28 \%$; desses, metade desenvolveu uma úlcera; $28,6 \%$, duas, e o restante $(21,4 \%)$, três. A média de UP por paciente foi de 1,7.

Quanto à localização das feridas, a região mais afetada foi a sacral $(57,1 \%)$, seguida pela calcânea (35,7\%), trocantérica (28,6\%) e ombro $(14,3 \%)$. Outras partes do corpo contribuíram com $28,6 \%$ das úlceras. O intervalo médio entre a internação e o aparecimento da primeira UP foi de 4,9 dias.

Avaliando a questão da presença de acompanhante durante a internação hospitalar, observou-se que tanto os indivíduos que desenvolveram UP quanto os que não apresentaram esse evento, em sua maioria, estavam com acompanhante (92,9\% e $75, \%$ respectivamente), sem diferença entre os grupos $(p=0,36)$.

As variáveis úlcera pregressa (sim/não), nível de atividade (acamado, dificuldade de deambular, sem restrições), uso de fraldas (número de dias de uso) e mudança de decúbito (número de dias que foi prescrita) estiveram associadas ao desenvolvimento de UP (Tabela 1).

Observou-se que a maioria dos indivíduos com prescrição de mudança de decúbito apresentava algum deficit de mobilidade $(94,1 \%$ contra $5,9 \%, p=0,004)$. Contudo, no grupo em que não

Tabela 1. Fatores associados à formação de úlceras de pressão. Belo Horizonte (MG), 2007.

\begin{tabular}{|c|c|c|c|c|c|}
\hline \multirow{3}{*}{ Variável } & \multicolumn{4}{|c|}{ UP (\%) } & \multirow{3}{*}{$p^{*}$} \\
\hline & \multicolumn{2}{|c|}{$\operatorname{sim}$} & \multicolumn{2}{|c|}{ Não } & \\
\hline & $\%$ & $\mathrm{n}$ & $\%$ & $n$ & \\
\hline \multicolumn{6}{|l|}{ úlcera pregressa } \\
\hline Sim & 21,4 & 3 & 5,6 & 2 & 0,13 \\
\hline Não & 78,6 & 11 & 94,4 & 34 & \\
\hline \multicolumn{6}{|l|}{ Nível de atividade } \\
\hline Acamado & 100,0 & 14 & 41,7 & 15 & $<0,001$ \\
\hline Dificuldade deambular & 0,0 & 0 & 44,4 & 5 & \\
\hline Sem restrições & 0,0 & 0 & 13,9 & 16 & \\
\hline \multicolumn{6}{|c|}{ Uso de fralda descartável (dias) } \\
\hline 0 & 7,1 & 1 & 50,0 & 18 & 0,002 \\
\hline 1 a 3 & 14,3 & 2 & 2,8 & 1 & \\
\hline 4 a 7 & 28,6 & 4 & 36,1 & 13 & \\
\hline 8 a 15 & 14,3 & 2 & 8,3 & 3 & \\
\hline$>15$ & 35,7 & 5 & 2,8 & 1 & \\
\hline \multicolumn{6}{|c|}{ Mudança de decúbito (dias) } \\
\hline 0 & 21,4 & 3 & 83,3 & 30 & $<0.001$ \\
\hline 1 a 3 & 21,4 & 3 & 0,0 & 0 & \\
\hline 4 a 7 & 28,6 & 4 & 8,3 & 3 & \\
\hline 8 a 15 & 7,1 & 1 & 8,3 & 3 & \\
\hline$>15$ & 21,4 & 3 & 0,0 & 0 & \\
\hline
\end{tabular}

* Qui-quadrado de Pearson ou teste exato de Fisher.

3 Salário-mínimo da época: $\mathrm{R} \$ 415,00$ 
foi recomendada a mudança de decúbito, 54,5\% dos pacientes eram acamados ou apresentavam dificuldade de deambulação.

Após classificar as morbidades segundo os capítulos do $\mathrm{CID}^{26}$, foram identificados 12 grupos de doenças. A medicação foi classificada segundo sua função ${ }^{27,28}$, obtendo-se 12 classes de medicamentos. Foi analisada a relação de ambos os grupos (doenças e medicação) com o desenvolvimento de úlceras (Tabela 2).

A maior prevalência das úlceras de pressão foi associada à presença de anemia, doença mental e do sistema nervoso. Quanto às demais morbidades (doenças do sistema circulatório, reumáticas, do aparelho respiratório, do sistema digestivo, infecciosas, metabólicas, oncológicas e urinárias), não foi verificada diferença estatisticamente significativa.

A UP foi mais frequente nos pacientes que receberam antibióticos $(p<0,008)$ e menos frequente nos que foram medicados com imunossupressores e anti-inflamatórios $(p<0,001)$ (Tabela 2). Quanto aos demais medicamentos (broncodilatadores, antiarrítmicos, anti-hipertensivos, an- tiangina, ansiolíticos, anticoagulantes, hipoglicemiantes e anti-histamínicos), não se observou diferença significativa entre os grupos $(p>0,05)$.

As variáveis antropométricas e os exames bioquímicos são apresentados na Tabela 3. No caso dos exames bioquímicos, os valores mostrados são referentes à média dos resultados dos exames que foram coletados a cada 30 dias de internação. Quanto às medidas antropométricas, foram avaliados os valores da admissão e da média de internação. As diferenças nos números de pacientes avaliados se devem: 1) ao fato de alguns não apresentarem resultados de exames de glicose; 2) à não aferição de pregas nos pacientes idosos em que a diferenciação entre o tecido adiposo e o muscular não foi possível; 3) à não aferição de CB e CP em pacientes que apresentavam edema de membros; e 4) à impossibilidade do cálculo da $A M B c$ em pacientes que não possuíam valores de PCT ou CB.

A Tabela 4 traz os dados referentes ao consumo alimentar dos pacientes e sua relação com a formação de UP. Três pacientes deixaram o estudo antes que fossem realizados os recordatórios 24 horas.

Tabela 2. Relação entre doenças e medicamentos e a formação de úlceras de pressão. Belo Horizonte (MG), 2007.

\begin{tabular}{|c|c|c|c|c|c|}
\hline \multirow{3}{*}{ Variável } & \multicolumn{4}{|c|}{ UP (\%) } & \multirow{3}{*}{$p^{*}$} \\
\hline & \multicolumn{2}{|c|}{ Sim } & \multicolumn{2}{|c|}{ Não } & \\
\hline & $\%$ & $\mathrm{n}$ & $\%$ & $\mathrm{n}$ & \\
\hline \multicolumn{6}{|l|}{ Anemia } \\
\hline Sim & 28,6 & 4 & 2,8 & 1 & 0,02 \\
\hline Não & 71,4 & 10 & 97,2 & 35 & \\
\hline \multicolumn{6}{|c|}{ Doença Mental } \\
\hline Sim & 42,9 & 6 & 11,1 & 4 & 0,02 \\
\hline Não & 57,1 & 8 & 88,9 & 32 & \\
\hline \multicolumn{6}{|c|}{ Doenças Sistema Nervoso } \\
\hline $\operatorname{sim}$ & 42,9 & 6 & 8,3 & 3 & 0,009 \\
\hline Não & 57,1 & 8 & 91,7 & 33 & \\
\hline \multicolumn{6}{|c|}{ Antibiótico } \\
\hline Sim & 85,7 & 12 & 44,4 & 16 & 0,008 \\
\hline Não & 14,3 & 2 & 55,6 & 20 & \\
\hline \multicolumn{6}{|c|}{ Antiinflamatório//munossupressor } \\
\hline Sim & 0 & 0 & 27,8 & 10 & $P<0,001$ \\
\hline Não & 100,0 & 14 & 72,2 & 26 & \\
\hline
\end{tabular}

* Qui-quadrado de Pearson ou teste exato de Fisher. 
Tabela 3. Relação entre indicadores bioquímicos e antropométricos e o aparecimento de úlceras de pressão. Belo Horizonte (MG), 2007.

\begin{tabular}{|c|c|c|c|c|c|c|}
\hline \multirow{3}{*}{ Variável ${ }^{1}$} & \multirow{3}{*}{$\mathrm{n}$} & \multicolumn{4}{|c|}{ UP } & \multirow{3}{*}{$p^{*}$} \\
\hline & & \multicolumn{2}{|c|}{ Sim } & \multicolumn{2}{|c|}{ Não } & \\
\hline & & M & DP & M & DP & \\
\hline Hemoglobina (g/dL) & 50 & 11,5 & 2,4 & 12,9 & 2,1 & 0,020 \\
\hline Hematócrito (\%) & 50 & 34,1 & 7,1 & 38,9 & 6,3 & 0,020 \\
\hline Leucócitos totais $\left(/ \mathrm{mm}^{3}\right)$ & 50 & 135,1 & 64,7 & 88,5 & 48,3 & 0,007 \\
\hline Plaquetas $\left(/ \mathrm{mm}^{3}\right)$ & 50 & 2209,6 & 1044,9 & 1862,7 & 814,0 & 0,290 \\
\hline Glicose & 33 & 149,6 & 57,2 & 148,6 & 69,9 & 0,670 \\
\hline \multicolumn{7}{|c|}{ Antropometria na internação } \\
\hline PCT & 34 & 5,7 & 2,3 & 14,3 & 5,5 & $<0,001$ \\
\hline PCB & 46 & 6,3 & 6,2 & 8,0 & 4,2 & 0,120 \\
\hline PCSE & 33 & 9,9 & 6,0 & 16,0 & 6,5 & 0,020 \\
\hline CB & 49 & 24,8 & 6,3 & 28,3 & 3,8 & 0,080 \\
\hline $\mathrm{AMBC}$ & 34 & 17,9 & 9,8 & 37,0 & 10,2 & 0,002 \\
\hline$C P$ & 48 & 26,8 & 4,6 & 32,9 & 3,9 & $<0,001$ \\
\hline \multicolumn{7}{|l|}{ Antropometria média² } \\
\hline PCT & 39 & 8,1 & 5,2 & 14,3 & 5,8 & 0,007 \\
\hline PCB & 49 & 6,9 & 5,7 & 7,9 & 4,6 & 0,280 \\
\hline PCSE & 35 & 9,3 & 5,8 & 15,8 & 6,5 & 0,008 \\
\hline CB & 50 & 25,2 & 5,9 & 28,1 & 3,7 & 0,110 \\
\hline $\mathrm{AMBC}$ & 39 & 21,9 & 11,3 & 37,1 & 10,4 & 0,004 \\
\hline $\mathrm{CP}$ & 50 & 27,5 & 5,2 & 32,8 & 3,7 & 0,002 \\
\hline
\end{tabular}

* Teste de Man-Whitney.

${ }^{1}$ Expressas como valores médios e desvio-padrão da população avaliada; ${ }^{2}$ Consiste nos valores médios, calculados a partir de todas as avaliações realizadas no período do estudo.

PCT: prega cutânea triciptal; PCB: prega cutânea biciptal; PCSE: prega cutânea subescapular; CB: circunferência do braço; AMBc: área muscular do braço corrigida; CP: circunferência de panturrilha.

Tabela 4. Associação de parâmetros dietéticos ao desenvolvimento de úlceras de pressão. Belo Horizonte (MG), 2007.

\begin{tabular}{|c|c|c|c|c|}
\hline \multirow{2}{*}{ Variável } & \multirow{2}{*}{$n$} & \multicolumn{2}{|c|}{ UP1 } & \multirow{2}{*}{$p^{*}$} \\
\hline & & Sim & Não & \\
\hline Nutrição Oral & 50 & & & $<0,001$ \\
\hline Sim & & 14,3 & 94,4 & \\
\hline Não & & 85,7 & 5,6 & \\
\hline Nutrição Enteral & 50 & & & $<0,001$ \\
\hline Sim & & 50,0 & 2,8 & \\
\hline Não & & 50,0 & 97,2 & \\
\hline Nutrição Oral/NE ${ }^{2}$ & 50 & & & \\
\hline Sim & & 28,6 & 0,0 & \\
\hline Não & & 71,4 & 100,0 & 0,004 \\
\hline Calorias & 47 & $1202,0 \pm 432,7$ & $1589,9 \pm 1429,7$ & 0,26 \\
\hline Proteínas & 47 & $55,1 \pm 23,0$ & $66,0 \pm 32,6$ & 0,29 \\
\hline Carboidratos & 47 & $147,1 \pm 54,9$ & $192,7 \pm 125,6$ & 0,21 \\
\hline Lípides & 47 & $45,0 \pm 18,2$ & $44,4 \pm 19,3$ & 0,97 \\
\hline Vitamina C. & 47 & $176,8 \pm 149,7$ & $49,3 \pm 31,8$ & $<0,001$ \\
\hline Zinco & 47 & $13,2 \pm 4,5$ & $7,5 \pm 3,5$ & $<0,001$ \\
\hline
\end{tabular}

${ }^{1}$ As variáveis nutrição oral, nutrição enteral e nutrição oral/nutrição enteral são apresentadas em valores percentuais. Para as demais são expostos os valores médios seguidos do desvio-padrão; ${ }^{2}$ Nutrição enteral.

* Qui-quadrado de Pearson ou teste exato de Fisher para as variáveis categóricas e teste de Man-Whitney para as contínuas. 
Tabela 5. Relação entre o desenvolvimento de úlceras de pressão, o motivo de desvinculação da pesquisa e o tempo de internação no hospital. Belo Horizonte (MG), 2007.

\begin{tabular}{lrrr}
\hline \multirow{2}{*}{ Variável } & \multicolumn{2}{c}{ UP $^{1}$} & \multirow{2}{*}{$p^{*}$} \\
\cline { 2 - 3 } & \multicolumn{1}{c}{$\operatorname{Sim}$} & Não & $<0,001$ \\
\hline Motivo de Saída do Estudo & & & \\
Alta & 21,4 & 80,0 & \\
Transferência & 7,1 & 8,6 & \\
Óbito & 50,0 & 8,6 & 0,12 \\
Fim do estudo & 21,4 & 2,8 & \\
Tempo de Internação & $16,9 \pm 11,7$ & $10,1 \pm 5,5$ & \\
\hline
\end{tabular}

${ }^{1}$ A variável Motivo de Saída do Estudo está expressa em valores percentuais. Já o Tempo de Internação é exposto como valor médio, em dias, seguido do desvio-padrão.

*Qui-quadrado para a variável categórica. Teste de Man-Whitney para a contínua.

Os dados que mostram a interferência das úlceras de pressão no tempo de permanência dos pacientes no hospital, assim como no motivo de sua desvinculação do estudo (Tabela 5).

\section{I S C U S S Ã O}

Na população estudada, verificou-se a predominância de indivíduos do sexo feminino, pardos ou negros, com a média de idade de $M=66,6$ $\mathrm{DP}=18,0$ anos (24-104 anos).

Apesar de o $\operatorname{sexo}^{8}$, a cor da pele e a idade 14-19,21-23,30 terem sido classificados por outros autores como fatores de risco para o desenvolvimento de úlceras, no presente trabalho não foi verificada associação estatisticamente significativa entre essas variáveis e o aparecimento de UP.

Blanes et al. ${ }^{13}$ caracterizam a população idosa como tendo uma maior suscetibilidade ao desenvolvimento de lesões devido às características causadas pelo envelhecimento da pele. $\mathrm{O}$ idoso apresenta aumento das comorbidades e do tempo de cicatrização, redução na elasticidade e textura da pele, na frequência de reposição celular, na mobilidade, além de diminuição da massa magra, levando à exposição das proeminências ósseas ${ }^{2}$.

Apesar da modernização dos cuidados de saúde, a prevalência das úlceras de pressão permanece elevada, particularmente nos doentes hospitalizados. Estudos anteriores mostram prevalências de UP, no ambiente hospitalar, variando entre $5,0 \%$ e $40,0 \%{ }^{4,13,15,18,20}$.

A incidência de UP nos pacientes internados na CLM e no CTI foi de $28 \%$. Sabe-se que pacientes do CTI podem ter grande risco para o desenvolvimento de úlceras, seja pela gravidade do quadro clínico, estado inflamatório e morbidades apresentadas, seja pelos baixos níveis de consciência e percepção sensorial, muitas vezes decorrentes de sedação ${ }^{6,8,13,14}$.

Encontrou-se certa dificuldade em avaliar os indivíduos internados no CTI devido à impossibilidade em assinar o termo de consentimento e muitas vezes à incompatibilidade dos horários de visita com os de coleta de dados.

As úlceras de pressão podem se desenvolver em 24 horas ou levar até 5 dias para sua manifestação ${ }^{31}$. No presente estudo, a primeira úlcera apareceu em média 4,9 dias após a admissão no hospital, aproximando-se do período encontrado por Costa ${ }^{2}$.

Alguns indivíduos desenvolveram mais de uma úlcera, sendo observado um máximo de três e uma média de 1,7 UP por paciente, sendo esse o mesmo valor encontrado por Costa et al. ${ }^{31}$.

As localizações mais frequentes foram a região sacral $(57,1 \%)$ e os calcanhares $(35,7 \%)$, que são áreas submetidas à grande pressão naqueles indivíduos que permanecem por longos períodos em decúbito dorsal, confirmando o descrito por outros autores ${ }^{2,5,11,19}$. Essas áreas são mais propensas à formação de UP devido à grande pressão exercida pelo peso corporal durante o repouso prolongado em superfície inadequada.

Além da pressão, considerada o principal fator etiológico da UP, a tolerância tecidual também tem papel importante na formação de úlceras. A exposição da pele à umidade, que pode ser exacerbada pela incontinência urinária e ou anal, é um fator que favorece a intolerância tissular 8,11,12,16,17,21,22. A maioria dos indivíduos do grupo com úlcera apresentou maior risco para exposição da pele à umidade, uma vez que 92,9\% utilizaram fralda em algum momento da internação $(p=0,002)$. 
O déficit de atividade mostrou ser estatisticamente significativo no desenvolvimento de UP, uma vez que $100,0 \%$ dos pacientes que desenvolveram UP estavam acamados, contra 41,6\% do grupo que não desenvolveu UP $(p<0,001)$.

A mudança de decúbito é uma importante recomendação presente na maioria dos protocolos de prevenção de UP, cuja orientação é que a mudança ocorra a cada duas horas. O objetivo dessa conduta é reduzir ou eliminar a pressão superficial e manter a microcirculação ${ }^{2,4,8,17}$.

Nesse trabalho a variável mudança de decúbito foi avaliada segundo o número de dias em que a medida estava prescrita no prontuário do paciente (Tabela 1). A maior parte dos indivíduos que apresentaram úlcera teve referência à mudança de decúbito em algum momento da internação $(78,6 \%)$; no grupo sem UP, somente para $16,6 \%$ houve tal prescrição $(p<0,001)$. Isso pode levar a dois questionamentos: 1) o deficit de mobilidade era menor entre pacientes que não desenvolveram UP, o que reduziu a necessidade da recomendação de reposicionamento; 2) a mudança de decúbito era prescrita como tratamento da UP, uma vez que estava significativamente mais presente entre os pacientes que desenvolveram a ferida.

Para responder a essas questões, inicialmente avaliou-se se havia relação entre a prescrição de mudança de decúbito e o grau de atividade dos indivíduos. Pode-se observar que, como esperado, a maior parte das prescrições era direcionada aos pacientes com algum déficit de mobilidade $(p=0,004)$. Contudo, por meio da análise inversa - quantos pacientes, no grupo com déficit de mobilidade, tinham prescrição de mudança de decúbito -, notou-se que mais da metade (54,5\%) não tinha registros dessa recomendação no prontuário.

Considerando que todos os pacientes que desenvolveram UP eram acamados e que a maioria das recomendações de mudança de decúbito estava concentrada nessa população, pode-se inferir que tal medida foi implementada como tratamento de úlceras e não como ação profilática.
A condição de saúde do paciente é um fator de extrema importância na avaliação do risco para o desenvolvimento de úlceras de pressão, já que a presença de determinadas morbidades pode implicar redução do nível de mobilidade e atividade (como doenças do sistema nervoso); interferir negativamente no fluxo sanguíneo e aporte de nutrientes e oxigênio para os tecidos (como doenças cardiorrespiratórias) ou ainda alterar a resposta imune do paciente, predispondo-o a infecções que podem agravar as condições de uma úlcera presente.

Várias doenças são citadas como fatores de risco para o desenvolvimento de UP. Dentre essas: diabetes mellitus descontrolado, lesão da medula espinhal, hipertensão arterial sistólica, sepse, anemia, infecção, doenças vasculares, res-

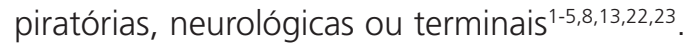

Entre os doze grupos de doenças diagnosticadas na amostra, somente anemias $(p=0,02)$, doenças mentais $(p=0,02)$ e doenças do sistema nervoso $(p=0,009)$ mostraram relação significativa com o desenvolvimento de UP. Hengstermann et al. ${ }^{16}$ e Souza \& Santos ${ }^{8}$ encontraram resultados semelhantes.

Problemas no sistema neurológico podem levar, entre outros eventos, à redução da locomoção e da percepção sensorial ${ }^{2}$. A anemia é comum em pessoas com UP e pode ser mais um reflexo de inflamação do que do estado nutricional ${ }^{32}$. Essas condições limitam a irrigação sanguínea e a mobilidade do paciente.

Quanto aos medicamentos utilizados, autores referem que alguns, geralmente de uso contínuo, embora necessários, podem contribuir para o desenvolvimento de UP. Os sedativos e analgésicos, por exemplo, reduzem a sensação de dor e prejudicam a mobilidade. Os agentes hipotensores, por outro lado, podem afetar o fluxo sanguíneo, reduzindo a perfusão dos tecidos e tornando-os mais susceptíveis à pressão 2,8,13,14.

Nesse estudo, somente a classe de antibióticos $(p=0,008)$ foi mais frequentemente prescrita para os indivíduos com UP, sendo seu uso significativo no que se refere ao desenvolvimento das feridas (Tabela 2). Por um lado, não há nenhum 
relato na literatura que indique que essa classe de medicamentos esteja envolvida na gênese da úlcera de pressão; por outro lado, eles podem ser indicativos de infecção, aspecto clínico frequente entre indivíduos com UP. O uso de anti-inflamatórios e imunossupressores foi mais frequente em pacientes que não desenvolveram UP.

Critérios antropométricos e bioquímicos de má-nutrição estão associados a um aumento da incidência e gravidade das úlceras de pressão ${ }^{20}$. Comparando os indicadores bioquímicos entre os dois grupos avaliados, notou-se que os pacientes com úlcera apresentaram menores médias de hemoglobina e hematócrito ( $p=0,02$ para ambos), dado importante uma vez que a baixa de ambos pode ser indicativa de anemia ${ }^{2}$. Esses resultados são fisiologicamente consistentes, já que o baixo nível desses indicadores implica menor capacidade de transporte de oxigênio e nutrientes aos tecidos, o que reduz sua viabilidade, predispondo à úlcera.

O organismo produz uma resposta inflamatória em reação às úlceras de pressão. Nessa resposta, observa-se o estímulo da proliferação de fibroblasto, mas também o aumento dos níveis de neutrófilos, que produzem proteases que destroem a fibronectina e dificultam o processo de cura da ferida ${ }^{11}$.

Foi observada associação estatisticamente significante entre maiores valores de leucócitos totais e desenvolvimento de UP $(p=0,007)$, o que pode ser representativo de maiores níveis de neutrófilos, implicando mau prognóstico das UP identificadas.

A desnutrição, além de aumentar o risco de úlceras, prejudica sua cura. Isso se deve à redução de nutrientes disponíveis para reparo e manutenção teciduais, resultando em perda do "efeito amortecedor" do tecido adiposo, menor resistência cutânea, fraqueza geral, mobilidade reduzida e edema ${ }^{1,32}$. Meijers et al. ${ }^{1}$ relatam que uma combinação de perda de massa magra e imobilidade aumenta o risco de UP para 74,0\%. Hengstermann et al. ${ }^{16}$, utilizando a Mini Avaliação Nutricional (MNA), verificaram que os escores dos pacientes com UP foram significativamente menores em comparação aos pacientes sem úlceras $(p=0,001)$, indicando pior estado nutricional. Langkamp-Henken et al. ${ }^{33}$ também utilizou a MNA para avaliar idosos institucionalizados que apresentavam UP, e observou que $86,9 \%$ dos indivíduos eram desnutridos ou estavam em risco de desnutrição. Esses autores encontraram uma correlação positiva entre as circunferências da panturrilha e do braço e a MNA. Rocha et al. ${ }^{20}$ citam os valores de prega tricipital inferiores a $3 \mathrm{~mm}$ nas mulheres e $2,5 \mathrm{~mm}$ nos homens como sendo indicadores antropométricos de má nutrição.

No presente trabalho, observou-se que os pacientes que desenvolveram úlcera tiveram valores significativamente menores de $A M B C, C P, P C T$, PCSE, assim como da soma dessas pregas, tanto na primeira avaliação, quanto nos valores médios (Tabela 3). Apesar de não ter sido calculado o percentual de adequação das medidas, pode-se inferir, a partir do exposto acima, que esse resultado indica maior fragilidade do estado nutricional dos pacientes que desenvolveram úlcera, estando essa condição presente desde a admissão e permanecendo ao longo da internação.

Quanto aos parâmetros dietéticos (Tabela 4), o grupo de pacientes com UP teve uma frequência significativamente maior do uso de suporte nutricional enteral parcial $(p<0,001)$ ou total $(p=0,004)$. Esse dado indica que esses indivíduos não tinham condição de se alimentar adequadamente pela via tradicional, isto é, oralmente, o que pode ser mais um indicador de debilidade clínica e nutricional.

Em termos de ingestão de nutrientes, observou-se consumo significativamente maior de vitamina $C$ e zinco pelos indivíduos que desenvolveram UP. Esse dado é consequência do fato de que a maioria desses pacientes estava usando dietas enterais industrializadas, que sabidamente têm maior teor de micronutrientes que as dietas convencionais.

A vitamina C e o zinco são descritos na literatura como importantes para cicatrização, regeneração, formação da pele e de tecidos de sustentação 2,3,6,11. Porém, o tempo de acompanhamento dos pacientes (máximo de 36 dias) não 
foi suficiente para mensurar o efeito desses micronutrientes sobre o desenvolvimento de UP.

Com relação à ingestão energética de macronutrientes, não houve diferença significativa entre os grupos, apesar de os pacientes com úlcera terem apresentado menores valores médios para proteína, carboidratos e calorias. Como os pacientes com UP mostraram piores médias antropométricas desde a internação (Tabela 3), a menor ingestão energética de macronutrientes possivelmente já ocorria antes mesmo da hospitalização, ou sua necessidade nutricional era maior devido ao estado nutricional e ao quadro clínico.

A UP está associada a um risco aumentado de mortalidade, que pode ser de 4 a 6 vezes maior em pacientes com a ferida ${ }^{31,32}$. No presente estudo, o desenvolvimento de UP também se associou significativamente a maiores índices de mortalidade $(50,0 \%$ contra $8,6 \%, p<0,001)$ (Tabela 5 ).

O tempo de internação é um fator de risco importante no que diz respeito ao desenvolvimento de UP, sendo significativo em alguns trabalhos ${ }^{2,16}$. Além disso, a presença de úlceras pode favorecer o aumento desse período de internação. Neste estudo, os pacientes que desenvolveram úlcera de pressão tiveram um tempo de internação 1,7 vezes maior que aqueles que não desenvolveram $(M=16,9, D P=11,7$ dias e $M=10,1$ $\mathrm{DP}=5,5$ dias, respectivamente) (Tabela 5). Entretanto, essa variável não apresentou significância estatística $(p=0,12)$.

\section{O N CLUS Ã O}

No presente estudo, o desenvolvimento de UP mostrou relação estatisticamente significativa com as variáveis, mudança de decúbito, nível de atividade, uso de fralda, presença de doenças mentais, neurológicas e anemia, assim como com o uso de antibióticos.

Observou-se também que um estado nutricional deficiente está estreitamente relacionado ao desenvolvimento de úlceras. Foram encontrados menores valores de tecido adiposo e massa muscular nos pacientes que desenvolveram UP, assim como baixos valores de hemo- globina e hematócrito, e valores elevados de leucócitos. Esses parâmetros nutricionais poderão ser ferramentas importantes para os profissionais no que diz respeito à identificação e ao tratamento de pacientes em risco.

Somente por meio da avaliação do risco para UP, do seu monitoramento e da atuação sobre os fatores que predispõem o desenvolvimento de úlceras, é possível reduzir a incidência desse evento, assim como os custos adicionais por ele gerados e, principalmente, o sofrimento do paciente.

Faz-se importante que os profissionais de saúde fiquem mais atentos aos pacientes que apresentam condições clínicas e nutricionais muito debilitadas desde a internação, pois, neste estudo, essa foi a população que mais desenvolveu UP.

\section{COLABORADORES}

A.B.P. COSTA, S.F. CAMPOS, A.P. CHAGAS e R. ESTEVÃO participaram da revisão bibliográfica, da concepção e do desenho do estudo A.K. JANSEN participou da redação do artigo.

\section{REFERÊ NCIAS}

1. Meijers JMM, Schols JMG, Jakson PA, Langer G, Clark M, Halfens RJG. Differences in nutritional care in pressure ulcer patients whether or not using nutritional guidelines. Nutrition. 2008; 24(2):127-32

2. Costa IG. Incidência de úlcera de pressão e fatores de risco relacionados em pacientes de um Centro de Terapia Intensiva [dissertação]. São Paulo: Universidade de São Paulo; 2003.

3. Desneves Kj, Todorovic BE, Cassar A, Crowe TC. Treatment with supplementary arginine, vitamin $C$ and zinc in patients with pressure ulcer: a randomized controlled trial. Clin Nutr. 2005; 24(6): 979-87.

4. Lewis M, Pearson A, Ward C. Pressure ulcer prevention and treatment: transforming research findings into consensus based clinical guidelines. J Nurs Pract. 2003; 9(2):92-102.

5. Uzun Ö, Tan M. A Prospective, descriptive pressure ulcer risk factor and prevalence study at a university hospital in Turkey. Ostomy Wound Manage. 2007; 53(2):44-56 
6. Theilla M, Singer P, Cohen J, Dekeyser F. A diet enriched in eicosapentanoic acid, gamma-linolenic acid and antioxidants in the prevention of new pressure ulcer formation in critically ill patients with acute lung injury: a randomized, prospective, controlled study. Clin Nutr. 2007; 26(6):752-7.

7. de Luis D, Aller R. Systematic review of nutritional support in pressure ulcer. An Med Interna. 2007; 24(7):342-5.

8. Souza DMST, Santos VLCG. Risk factors for pressure ulcer development in institutionalized elderly. Rev Lat-Am Enfermagem. 2007; 15(5):958-64.

9. National Pressure Ulcer Advisory Panel. Pressure ulcer prevalence, cost and risk assessment: consensus development conference statement. Decubitus. 1989; 2(2):24-8.

10. European Pressure Ulcer Advisory Panel. Pressure Ulcer Treatment Guidelines. Oxford: advisory panel. [cited 2008 Jul 2]. Available from: <http://www. epuap.org/gltreatment.html>

11. Gomes FSL, Magalhães MBB. Úlcera por pressão. In: Borges EL, Saar SRC, Lima VLAN, Gomes FSL, Magalhães MBB. Feridas: como tratar. $2^{\mathrm{a}}$ ed. Belo Horizonte: Coopmed; 2007. p.189-223

12. Cunha DF, Frota RB, Arruda MS, Cunha da SFC, Teixeira VPA. Pressure sores among malnourished necropsied adults: preliminary data. Rev Hosp Clin Fac Med São Paulo. 2000; 55(3):79-82.

13. Blanes L, Duarte IS, Calil JA, Ferreira LM. Avaliação clínica e epidemiológica das úlceras por pressão em pacientes internados no Hospital São Paulo. Rev Assoc Med Bras. 2004; 50(2):182-7.

14. Rogenski NMB, Santos VLCG. Estudo sobre a incidência de úlceras por pressão em um hospital universitário. Rev Lat-Am Enfermagem. 2005; 13(4): 474-80.

15. Pedro-Hidalgo PL, Garcia-Fernando FP, López-Medina IM, López-Ortega J. Pressure ulcer care in Spain: nurses' knowledge and clinical practice. J Adv Nurs. 2007; 58(4):327-38.

16. Hengstermann S, Fischer A, Steinhagen-Thiessen E, Schulz R. Nutrition status and pressure ulcer: what we need for nutrition screening. J Parenter Enteral Nutr. 2007; 31(4):288-94.

17. Reddy M, Gill SS, Rochon PA. Preventing pressure ulcers: a systematic review. J Am Med Assoc. 2006; 296(8):974-84.

18. Harris CL, Fraser C. Malnutrition in the institutionalized elderly: the effects on wound healing. Ostomy Wound Manage. 2004; 50(10):54-63.

19. Cuddigan J, Berlowitz DR, Ayello EA. Pressure ulcers in America: prevalence, incidence, and implications for the future. Adv Skin Wound Care. 2001; 14(4): 208-15.
20. Rocha JA, Miranda MJ, Andrade MJ. Abordagem terapêutica das úlceras de pressão - intervenções baseadas em evidências. Acta Med Port. 2006; 19:29-38.

21. Schols JMGA, Ende MAJ. Nutritional intervention in pressure ulcer guidelines: an inventory. Nutrition. 2004; 20(6):548-53,

22. Nogueira PC, Caliri MHL, Santos CB. Fatores de risco e medidas preventivas para úlcera de pressão no lesado medular. Experiência da equipe de enfermagem do HCFMRP-USP. Medicina (Ribeirão Preto). 2002; 35(1):14-23.

23. Ayello EA, Braden B. How and why to do pressure ulcer risk assessment. Adv Skin Wound Care. 2002; 15(3):125-33.

24. World Health Organization. Physical status: the use and interpretation of anthropometry. Geneva: WHO; 1995. Technical Report, Series 854.

25. Menezes TN, Marucci MFN. Perfil dos indicadores de gordura e massa muscular corporal dos idosos de Fortaleza, Ceará, Brasil. Cad Saúde Pública. 2007; 23(12):2887-95.

26. Organização Mundial da Saúde. Manual da classificação estatística internacional de doenças, lesões e causas de óbito. São Paulo: USP; 1985.

27. Brasil.Portaria $n^{\circ}$ 1.587. Agência Nacional de Vigilância Sanitária. 2002.

28. Martins C, Moreira SM, Pierosan SR. Interação droga nutriente. $2^{a}$ ed. Curitiba: Nutroclínica; 2003.

29. Núcleo de Estudos e Pesquisas em Alimentação. Tabela brasileira de composição dos alimentos. $2^{\text {a }}$ versão. Campinas: Unicamp; 2006.

30. Rocha ABL, Barros SMO. Avaliação de risco de úlcera por pressão: propriedades de medida da versão em português da escala Waterlow. Acta Paul Enferm. 2007; 20(2):143-50.

31. Costa MP, Sturtz G, Costa FPP, Ferreira MC, Filho TEPB. Epidemiologia e tratamento das úlceras de pressão: experiência de 77 casos. Acta Ortop Bras 2005; 13(3):124-33.

32. Stratton RJ, Ek AC, Engfer M, Moore Z, Rigby $P$, Wolfe $R$, et al. Enteral nutritional support in prevention and treatment of pressure ulcers: A systematic review and meta-analysis. Ageing Res Rev. 2005; 4(3):422-50.

33. Langkamp-Henken B, Hudgens J, Stechmiller JK, Herrlinger-Garcia KA. Mini nutritional assessment and screening scores are associated with nutritional indicators in elderly people with pressure ulcers. J Am Diet Assoc. 2005; 105(10):1590-96.

Recebido em: 3/11/2008 Versão final reapresentada em: 29/10/2009 Aprovado em: 4/5/2010 